\title{
Fronts between hexagons and squares in a generalized Swift-Hohenberg equation
}

\author{
Kubstrup, Christian; Herrero, H.; Pérez-García, C.
}

Published in:

Physical Review E. Statistical, Nonlinear, and Soft Matter Physics

Link to article, DOI:

10.1103/PhysRevE.54.1560

Publication date:

1996

Document Version

Publisher's PDF, also known as Version of record

Link back to DTU Orbit

Citation (APA):

Kubstrup, C., Herrero, H., \& Pérez-García, C. (1996). Fronts between hexagons and squares in a generalized Swift-Hohenberg equation. Physical Review E. Statistical, Nonlinear, and Soft Matter Physics, 54(2), 1560-1569. https://doi.org/10.1103/PhysRevE.54.1560

\section{General rights}

Copyright and moral rights for the publications made accessible in the public portal are retained by the authors and/or other copyright owners and it is a condition of accessing publications that users recognise and abide by the legal requirements associated with these rights.

- Users may download and print one copy of any publication from the public portal for the purpose of private study or research.

- You may not further distribute the material or use it for any profit-making activity or commercial gain

- You may freely distribute the URL identifying the publication in the public portal 


\title{
Fronts between hexagons and squares in a generalized Swift-Hohenberg equation
}

\author{
C. Kubstrup, ${ }^{1}$ H. Herrero, ${ }^{2}$ and C. Pérez-García ${ }^{2}$ \\ ${ }^{1}$ Center for Chaos and Turbulence Studies, Physics Department, Technical University of Denmark, DK-2800 Lyngby, Denmark \\ ${ }^{2}$ Departamento de Física y Matemática Aplicada, Facultad de Ciencias, Universidad de Navarra, E-31080 Pamplona, Navarra, Spain
}

(Received 3 February 1995; revised manuscript received 15 September 1995)

\begin{abstract}
Pinning effects in domain walls separating different orientations in patterns in nonequilibrium systems are studied. Usually, theoretical studies consider perfect structures, but in experiments, point defects, grain boundaries, etc., always appear. The aim of this paper is to perform an analysis of the stability of fronts between hexagons and squares in a generalized Swift-Hohenberg model equation. We focus the analysis on pinned fronts between domains with different symmetries by using amplitude equations and by considering the small-scale structure in the pattern. The conditions for pinning effects and stable fronts are determined. This study is completed with direct simulations of the generalized Swift-Hohenberg equation. The results agree qualitatively with recent observations in convection and in ferrofluid instabilities. [S1063-651X(96)10707-8]
\end{abstract}

PACS number(s): 47.27.Te, 47.54.+r, 44.25.+f

\section{INTRODUCTION}

Pattern formation is quite common in fluids, lasers, and chemical reactions, where a huge amount of research work is being done. Most efforts have been addressed to a universal understanding of these phenomena, where notable advances have been accomplished [1]. One of the topics that received attention is the dynamics of defects and of fronts in these nonequilibrium systems. Stationary fronts have been established in the transition between roll and hexagonal patterns in convection under non-Boussinesq conditions. The competition between hexagons and squares has been theoretically analyzed [2,3] and experimentally observed [4] in interfacial instabilities of ferrofluids under a vertical magnetic field [5]. Just recently such a competition has also been observed in Bénard-Marangoni convection [6,7]. This competition can lead to interesting features: defects, grain boundaries, hysteretic effects, etc. In the present paper we will analyze the stability conditions for fronts between square and hexagonal structures. We will follow the outlines of Refs. [8,9], where this specific problem was only partially solved.

In the vicinity of an instability threshold, a systematic procedure allows us to reduce the evolution equation to linearly unstable modes (center manifold reduction). Spatial variations can also be included leading to generalized Ginzburg-Landau equations (GLE's) for the amplitude of those modes $[10,11]$. However, this formalism is limited to a finite number of spatial Fourier modes, since each mode requires an extra envelope equation. Swift and Hohenberg [12] proposed a model equation for a rapidly varying scalar function. This equation reflects, mainly, effects of a rotationally invariant linear selection. It is possible to generalize this model to include spatial derivatives in the nonlinearities that can lead to square and hexagonal patterns [13]. We shall refer to this generalized Swift-Hohenberg equation as GSHE. This GSHE allows for numerical simulations that resemble quite well the patterns found in several nonequilibrium systems.

The main aim of this paper is to present theoretical results on pinned fronts between patches of different symmetries in patterns where hexagonal and square cells coexist. In the first section we discuss a minimal GSHE model to obtain those kinds of patterns. As analytical studies on the GSHE are quite difficult, we proceed in several steps. We derive the amplitude equations for this GSHE model and we quote the stability of the stationary solutions under spatially homogeneous perturbations. With these amplitude equations, the value of the control parameter that leads to an immobile (pinned) front between hexagons and squares can be obtained. This parameter value must correspond to a solution in the hysteric region where both hexagons and squares are stable, and its derivation is performed in Sec. II. Using this reference value, we shall use the GSHE to show that the pinning effect is possible for a whole interval of parameter values, due to the interplay between the small-scale structure and the envelope field (nonadiabatic effects) [14]. An estimation of this interval is given in Sec. III.

Numerical simulations of the GSHE are presented in Sec. IV, thereby checking analytical results and evaluating the constants for which calculations in previous sections only give the order. We will bring phenomenological results that support the grouping of the fronts in two different types. Section $\mathrm{V}$ is devoted to a discussion of the results and to present the main conclusions.

\section{GENERALIZED SWIFT-HOHENBERG EQUATION}

The Swift-Hohenberg equation was introduced to study patterns of rolls in Rayleigh-Bénard convection [12]. Bestehorn and co-workers [15-17] have systematically generalized this equation to include more general planforms. The GSHE can be thought of as an approximation in real space to an order-parameter equation that is possible to deduce from the basic equations. A GSHE that allows haxagons and squares to evolve, takes the form $[17,18,9]$ :

$$
\dot{\psi}=\left[\varepsilon-(1+\Delta)^{2}\right] \psi-\alpha \psi^{2}-\beta \psi^{3}+\delta \psi \Delta \psi^{2}-\gamma \psi \Delta^{2} \psi^{2} .
$$

This is an equation for a real-order parameter $\psi(r, t)$, a scalar variable proportional to the velocity and temperature fields in convection or to deviations of the interface with 
respect to the midplane in ferrofluids; $\Delta$ is the Laplacian in two dimensions. Here $\varepsilon$ is the control parameter defined as a distance to criticality. We simplify further this last equation keeping only the essential terms: one which makes the hexagonal symmetry possible and another responsible for the squares. Then we propose the following equation as a minimal model:

$$
\dot{\psi}=\left[\varepsilon-(1+\Delta)^{2}\right] \psi+\alpha \psi^{2}-\beta \psi^{3}-\gamma \psi \Delta^{2} \psi^{2} .
$$

When $\alpha=\gamma=0$ we recover the Swift-Hohenberg equation discussed in Ref. [12]. From the linear stability analysis we find that $\varepsilon=O(\epsilon)$ and we assume that $\alpha=O(\sqrt{\epsilon})$. Usually the following boundary conditions (BC's) are assumed,

$$
\psi=\partial_{\mathbf{n}} \psi=0,
$$

where $\mathbf{n}$ is a unit vector normal to the sidewalls.

\section{A. Stability analysis under spatially homogeneous perturbations}

We now consider situations where the system develops almost perfect patterns with a well-defined symmetry. The scalar variable $\psi$ can be developed in terms of plane waves that can lead to rolls, squares, or hexagons:

$$
\psi(\mathbf{x}, t)=\sum_{j=1}^{6}\left[A_{j}\left(X_{j}, Y_{j}, T\right) e^{i \mathbf{k}_{j} \cdot \mathbf{x}}+\text { c.c. }\right]+O\left(A_{i}^{2}\right),
$$

where $X_{j} \equiv \mathbf{X} \cdot \mathbf{n}_{j}, Y_{j} \equiv \mathbf{X} \cdot \tau_{j}$, and $\mathbf{n}_{j}$ and $\tau_{j}$ are mutually orthogonal unit vectors. As usual, two temporal and spatial scales can be distinguished in the system: for the envelope function $\mathbf{X}$ and $T$ are the slow variables, and $\mathbf{x}, t$ are the fast variables for the underlying structure [19]. $A_{j}(T)$, $j=1,2, \ldots, 6$ are the amplitudes of the modes with wave vectors $\mathbf{k}_{j}, j=1,2, \ldots, 6$, corresponding to the directions of the six principal modes [1]. These wave vectors are related by $\mathbf{k}_{1}+\mathbf{k}_{2}+\mathbf{k}_{3}=\mathbf{0}, \mathbf{k}_{4}+\mathbf{k}_{5}+\mathbf{k}_{6}=\mathbf{0}, \mathbf{k}_{j} \cdot \mathbf{k}_{j+3}=0, j=1,2,3$, and $\left|\mathbf{k}_{j}\right|=k_{c}$. Here six orientations are included in order to allow for two conjugated sets of hexagons and structures containing both hexagons and squares (see Fig. 2 and further explanations in the text). After introducing this development into Eq. (2) one obtains a set of amplitude equations:

$$
\begin{aligned}
\dot{A}_{1}= & \varepsilon A_{1}+4\left[\frac{\partial}{\partial X_{1}}-\frac{i}{2 k_{c}} \frac{\partial^{2}}{\partial Y_{1}^{2}}\right]^{2} A_{1}+a \overline{A_{2}} \overline{A_{3}} \\
& -\left[b\left(\left|A_{2}\right|^{2}+\left|A_{3}\right|^{2}\right)+c\left|A_{1}\right|^{2}+d\left(\left|A_{5}\right|^{2}+\left|A_{6}\right|^{2}\right)\right. \\
& \left.+e\left|A_{4}\right|^{2}\right] A_{1}, \\
\dot{A}_{4}= & \varepsilon A_{4}+4\left[\frac{\partial}{\partial X_{4}}-\frac{i}{2 k_{c}} \frac{\partial^{2}}{\partial Y_{4}^{2}}\right]^{2} A_{4}+a \overline{A_{5}} \overline{A_{6}} \\
& -\left[b\left(\left|A_{5}\right|^{2}+\left|A_{6}\right|^{2}\right)+c\left|A_{4}\right|^{2}+d\left(\left|A_{2}\right|^{2}+\left|A_{3}\right|^{2}\right)\right. \\
& \left.+e\left|A_{1}\right|^{2}\right] A_{4},
\end{aligned}
$$

the rest are obtained by cyclic permutations of the indices, indices 1,2,3, and 4,5,6. The BC's, are

$$
A=\partial_{n} A=0,
$$

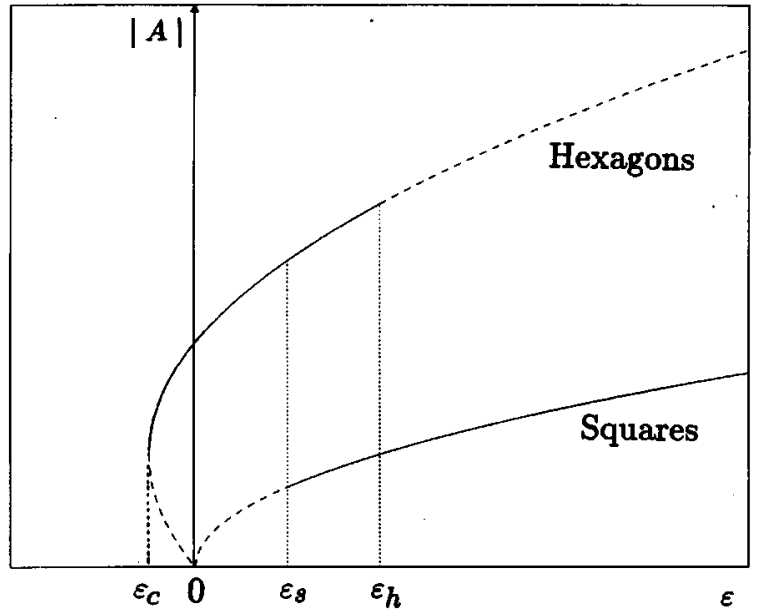

FIG. 1. Stability of the hexagons and squares, as a function of $\varepsilon$. Note the hysteretic region for $\varepsilon_{s} \leqslant \varepsilon \leqslant \varepsilon_{h}$.

but we assume in the following that the system has an infinite extent. In fact, these equations are the amplitude equations of the GSHE, provided we identify the coefficients:

$$
\begin{gathered}
a=2 \alpha, \quad b=6 \beta+20 \gamma, \quad c=3 \beta+16 \gamma, \\
d=6 \beta+28 \gamma, \quad e=6 \beta+16 \gamma .
\end{gathered}
$$

Now we are interested in the stability threshold for each symmetry and not for a full stability analysis of that system. Then we restrict the linear stability analysis to spatially homogeneous perturbations around stationary solutions. (The stability analysis for nonhomogeneous perturbations can also be performed $[20,21])$. The results were obtained and discussed in previous works $[8,23]$. In summary, squares are stable if

$$
\beta<0 \wedge \varepsilon>\frac{4 \alpha^{2}(9 \beta+32 \gamma)}{(3 \beta+16 \gamma)^{2}}=\varepsilon_{s}>0,
$$

and hexagons are stable if

$$
\varepsilon_{c}=\frac{-\alpha^{2}}{15 \beta+56 \gamma}<\varepsilon<\frac{16 \alpha^{2}(3 \beta+13 \gamma)}{(3 \beta+4 \gamma)^{2}}=\varepsilon_{h} .
$$

(We present these results in terms of parameters in the GSHE for the sake of comparison with numerical simulations.) A graphical scheme of the above results is given in Fig. 1. Stability for hexagons requires a further condition, namely that $|(15 \beta+56 \gamma) /(18 \beta+72 \gamma)| \leqslant 1$. (We will show that this condition is fulfilled in our case, but generally this may not be so.) This extra condition is a result of including perturbations perpendicular to the hexagonal modes.

So far we have only considered hexagonal and square patterns. From the form of the amplitude equations one can deduce that the hexagonal pattern formed by a resonant triad is independent of other interacting modes [22]. (Mixed modes as, for example, $A_{1}=A_{2} \neq A_{3}$ and $A_{4}=A_{5}=A_{6}=0$ are always unstable in a system displaying hexagons.) It can furthermore be shown that the roll pattern never is stable in a system permitting a stable square pattern. Further analysis shows also that rectangular as well as rhombic patterns are 
impossible or always unstable; in other words, the amplitude equations do not provide planforms for any more of the usual structures to be stable.

\section{CONDITIONS FOR A FRONT}

An important property of the amplitude equations is that they are relaxational, i.e., they can be deduced from a Lyapunov functional $F[A]$ :

$$
\begin{aligned}
F[A]= & \int d X d Y \sum_{j=1}^{6}\left[-\varepsilon\left|A_{j}\right|^{2}+\frac{c}{2}\left|A_{j}^{2}\right|^{4}\right. \\
& \left.+4\left|\left(\frac{\partial}{\partial X_{j}}-\frac{i}{2 k_{c}} \frac{\partial^{2}}{\partial Y_{j}^{2}}\right) A_{j}\right|^{2}\right]+\sum_{j, l, j \neq l}^{6} g_{j l} \frac{c}{2}\left|A_{j}\right|^{2}\left|A_{l}\right|^{2} \\
& -a\left(\overline{A_{1}} \overline{A_{2}} \overline{A_{3}}+\overline{A_{4}} \overline{A_{5}} \overline{A_{6}}\right),
\end{aligned}
$$

where $g_{j l}$ are the coupling coefficients seen in (5). The minima of this functional correspond to the stationary states, and $A_{j}$ is a solution of

$$
\dot{A}_{j}=-\frac{\delta F[A]}{\delta \overline{A_{j}}},
$$

where $\delta$ is a Fréchet derivative. For many values of the parameter, the functional has several local minima, all corresponding to stable solutions. The comparison between the energies of the minima of $F[A]$ allows for a prediction of towards which state the system is likely to evolve, but the actual evolution depends on the initial conditions.

To make the algebra more tractable we will consider from now on primarily one spatial variable $x$ (assumed to be perpendicular to the front) and we average in the $y$ coordinate. We rescale the amplitude equations in the usual way. (After the averaging, all $y$-dependence can be taken as zero):

$$
\begin{gathered}
A_{j}=\frac{a}{c} a_{j}, \quad T^{\prime}=\frac{a^{2}}{c} T, \quad X^{\prime}=\frac{a}{2 \sqrt{c}} X, \quad D_{j}=\left(k_{j}\right)_{x}^{2}, \\
g_{1}=\frac{b}{c}, \quad g_{2}=\frac{d}{c}, \quad g_{3}=\frac{e}{c}, \quad \delta=\frac{\varepsilon c}{a^{2}} .
\end{gathered}
$$

Notice that now the control parameter is $\delta$. Stationary solutions of hexagons, where $a_{h}=\left[1+\sqrt{1+4 \delta\left(1+2 g_{1}\right)}\right] /$ $\left[2\left(1+2 g_{1}\right)\right]$ and stationary squares $a_{s}=\sqrt{\delta /\left(1+g_{2}\right)}$, have the following stability intervals for the homogeneous perturbations in this notation [see Eqs. (8) and (9)]:

$$
\begin{gathered}
\delta>\frac{1+g_{2}}{\left(1-g_{1}+g_{2}-g_{3}\right)^{2}} \text { for squares, } \\
\frac{-1}{4\left(1+2 g_{1}\right)} \leqslant \delta \leqslant \frac{g_{1}+2}{\left(g_{1}-1\right)^{2}} \text { for hexagons, }
\end{gathered}
$$

with the extra condition $\left|\left(2 g_{1}+1\right) /\left(2 g_{2}+g_{3}\right)\right| \leqslant 1$.

To study a mobile-front solution with constant velocity $c$, we write $a_{j}$ as $a_{j}(\xi), \xi=X^{\prime}-c T^{\prime}$. The functions $a_{j}(\xi)$ are then determined by the equations

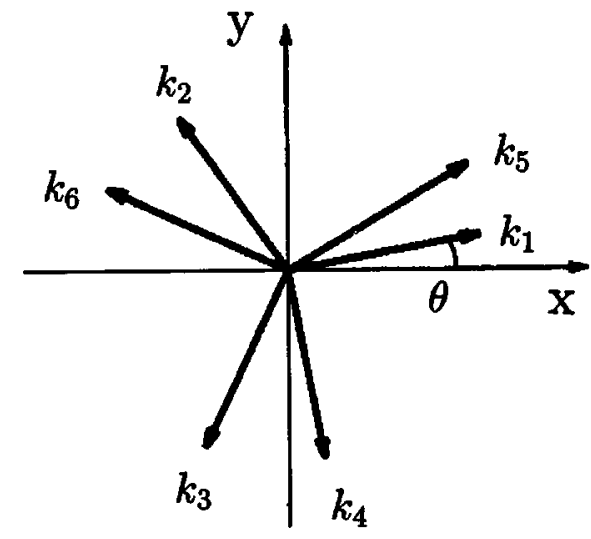

FIG. 2. The six modes considered in the study shown with the angle $\theta$ as mentioned in the text. 1,2,3 and 4,5,6 form resonant triads and 1 and 4 are perpendicular.

$$
\begin{aligned}
D_{1} a_{1}^{\prime \prime} & +c a_{1}^{\prime}+\delta a_{1}+a_{2} a_{3}-a_{1}\left[a_{1}^{2}+g_{1}\left(a_{2}^{2}+a_{3}^{2}\right)+g_{2} a_{4}^{2}\right. \\
& \left.+g_{3}\left(a_{5}^{2}+a_{6}^{2}\right)\right]=0, \\
D_{4} a_{4}^{\prime \prime} & +c a_{4}^{\prime}+\delta a_{4}+a_{5} a_{6}-a_{4}\left[a_{4}^{2}+g_{1}\left(a_{5}^{2}+a_{6}^{2}\right)+g_{2} a_{1}^{2}\right. \\
& \left.+g_{3}\left(a_{2}^{2}+a_{3}^{2}\right)\right]=0,
\end{aligned}
$$

with the BC's

$$
\begin{gathered}
\left|a_{1}\right|=\left|a_{2}\right|=\left|a_{3}\right|=a_{h} \neq 0, \quad a_{4}=a_{5}=a_{6}=0 \quad \text { for } x=-\infty, \\
\left|a_{1}\right|=\left|a_{4}\right|=a_{s} \neq 0, \quad a_{j}=0, \quad j=2,3,5,6 \quad \text { for } x=\infty,
\end{gathered}
$$

where

$$
\begin{gathered}
D_{1}=\cos ^{2} \theta, \quad D_{2}=\frac{1}{4}(\cos \theta-\sqrt{3} \sin \theta)^{2}, \\
D_{3}=\frac{1}{4}(\cos \theta+\sqrt{3} \sin \theta)^{2}, \\
D_{4}=\sin ^{2} \theta, \quad D_{5}=\frac{1}{4}(\sqrt{3} \cos \theta+\sin \theta)^{2}, \\
D_{6}=\frac{1}{4}(\sqrt{3} \cos \theta-\sin \theta)^{2},
\end{gathered}
$$

$D_{j}$ are in units of $k_{c}=1$, and $\theta$ is the angle between $\mathbf{k}_{1}$ and a vector normal to the front (see Fig. 2 where the front is assumed to be vertical). The stationary versions of Eqs. (16) $(c=0)$ can be considered as the equation of motion of a mechanical system with a Lagrangian $L$ :

$$
\begin{gathered}
L=\sum_{j=1}^{6} \frac{1}{2} D_{j}\left(a_{1}^{\prime}\right)^{2}-V \\
V=\sum_{j=1}^{6}\left(\frac{1}{2} \delta a_{j}^{2}-\frac{1}{4} a_{j}^{4}\right)-\sum_{j, l, j<l} \frac{g_{j l}}{2} a_{j}^{2} a_{l}^{2}+a_{1} a_{2} a_{3}+a_{4} a_{5} a_{6} .
\end{gathered}
$$

An immobile front corresponds to a trajectory of the mechanical system joining two local maxima of the effective potential $V$, which corresponds to two different structures. A maximum of the effective potential corresponds to a minimum of $L$ and of the Lyapunov functional $F[A]$. This struc- 

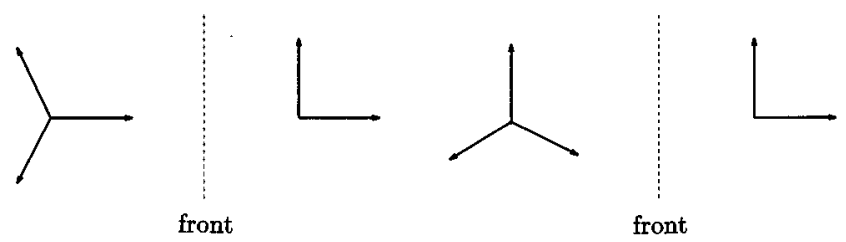

FIG. 3. Fronts of types I and II, respectively.

ture can exist only if the values of the potential $V$ in both points are equal. The front moves to the "right" if $L(-\infty)$ $>L(\infty)$, i.e., the structure with the lower value of $F[A]$ invades the one with the larger value. For two given homogeneous stationary structures, there is a unique value of the control parameter $\delta_{1}$ that fulfils this condition. Usually this value can be found only numerically [9], but it can be given analytically in some particular cases considered in the following.

Two extreme cases of possible orientation in the front can be considered. A scheme of these two types of fronts is shown in Fourier space in Fig. 3. These two fronts are the simplest to describe, but fronts with arbitrary angles $\theta$ can also be studied. Type I corresponds to fronts with $\theta$ close to zero, and type II to those with $\theta$ different from zero. The calculations will only be performed for the type-I front $(\theta$ $=0$ ), since for the type-II front the short-scale effects eliminate the possibility of pinning.

An analytical solution can be found for $0<g_{1}-1 \ll 1$, small $\delta-\delta_{1}$ and $g_{2}-1$, as well as setting $g_{3}=\frac{3}{4}+g_{2}$ in Eqs. (16). If $\theta=0$, then $D_{2}=D_{3}$, and there is a solution with $a_{2}=a_{3}$ and $a_{5}=a_{6}=0$. In this case, a front between the solutions $a_{4}=a_{5}=a_{6}=0, a_{1} \neq 0, a_{2}=a_{3} \neq 0$ at $x \rightarrow-\infty$, and $a_{1}=a_{4} \neq 0, a_{2}=a_{3}=a_{5}=a_{6}=0$ at $x \rightarrow \infty$ can stabilize if the potential given in expression [21] obeys $|V(-\infty)-V(\infty)|$ $=0$. This condition is fulfilled for the following value of the control parameter $\delta$ :

$$
\delta=\left(4 / 3 g_{2}+\frac{2}{3}\right)\left(g_{1}-1\right)^{-2} .
$$

(The detailed calculations are given in Appendix A.)

\section{A. Nonadiabatic pinning effects}

The possibility of a stable front has been determined by amplitude equations in Sec. II. We determined the value of the control parameter to have such a front with a condition similar to the Maxwell construction in phase transitions. But this value is not exact because it does not take into account small-scale effects. When these effects are included, $\mathbf{x}$ and $\mathbf{X}$ are no longer independent, giving rise to exponentially small (nonadiabatic) effects. In Ref. [14] it has been shown that interaction of a mobile front with these small-scale structures can give rise to the pinning of the front with an adjusting wave-number field in the pattern. In the case of hexagons vs rolls, it has furthermore been shown that if the front is almost perpendicular to one of the wave vectors $\mathbf{k}_{j}$ of the hexagons, a pinning is possible without requiring a change in the underlying wave number [8].

The GSHE includes the two scales and is therefore a suitable model for a study of the full interval for pinning effects. With the rescaled coefficients the GSHE reads as

$$
\dot{\psi}=\left[\epsilon \delta-(1+\Delta)^{2}\right] \psi+\alpha \psi^{2}-\frac{\beta}{3} \psi^{3}-\gamma \psi \Delta^{2} \psi^{2}
$$

A front between hexagons and squares can be described as a scalar field:

$$
\psi=2 \sqrt{\epsilon} \sum_{j=1}^{4} a_{j} \cos \mathbf{k}_{j} \cdot \mathbf{x}+O(\epsilon),
$$

where the amplitudes $a_{j}$ are solutions of the rescaled Ginzburg-Landau (GL) equation (16) when $\theta=0$. In this case, we have $D_{1}=1, D_{2}=D_{3}=\frac{1}{4}, D_{4}=0, D_{5}=D_{6}=\frac{3}{4}$, and $a_{2}=a_{3}$ The front does not move for $\delta \equiv \delta_{1} \gg 1$.

Of course the last equation is not an exact solution of the GSHE, even though the amplitudes were exact solutions of the GL equations (5). We are looking for a corrected solution (at lowest order) for small $\left|\delta-\delta_{1}\right|$, such that

$$
\psi=\psi_{0}+\bar{\psi},
$$

where

$$
\psi_{0}=2 \sqrt{\epsilon} \sum_{j=1}^{4} a_{j}(\sqrt{\epsilon}[x-\xi(\epsilon t)]) \cos _{j} \cdot x,
$$

and $\widetilde{\psi}$ has its origin in nonadiabatic effects and in the small differences $\left|\delta-\delta_{1}\right|$, i.e., $\widetilde{\psi}=O(\epsilon)$.

A linear perturbative analysis around the solution $\psi_{0}$ gives the following linearized equation for $\widetilde{\psi}$ :

$$
\begin{gathered}
\delta_{1} \widetilde{\psi}-(1+\Delta)^{2} \widetilde{\psi}+2 \alpha \psi_{0} \tilde{\psi}-\beta \psi_{0}^{2} \widetilde{\psi}-\gamma 2 \psi_{0} \Delta^{2}\left(\psi_{0} \widetilde{\psi}\right) \\
-\gamma \widetilde{\psi} \Delta^{2} \psi_{0}^{2}=\frac{\partial \psi_{0}}{\partial \xi} \frac{d \xi}{d t}-\left(\delta-\delta_{1}\right) \psi_{0}-G,
\end{gathered}
$$

where $G$ contains the fast dependence on $\mathbf{x}$ in $\cos \left(\mathbf{k}_{j} \cdot \mathbf{x}\right)$ that is the source of nonadiabaticity. Explicit calculation of $G$ is too cumbersome a task to be included here. We include an outline of this calculation in Appendix B.

The main result of the calculation that we get from (B10) is the following estimate of the interval around $\delta_{1}$ for which the front between hexagons and squares is immobile:

$$
\left|\delta-\delta_{1}\right|<C_{3} \exp \left[-\frac{\sqrt{3}-1}{8} \pi\left(\frac{3\left(g_{1}-1\right)}{\epsilon}\right)^{1 / 2}\right],
$$

where $C_{3}$ is a constant. This means that a front between squares and hexagons is observable for this full range of the control parameter. It is important that, close to the critical value $g_{1}=1, \epsilon \ll g_{1}-1 \ll 1$, since in this case the fact of $\epsilon$ being small may be compensated by $g_{1}-1$. In the limit $\epsilon \simeq g_{1}-1$, the interval of pinning is not exponentially small and the analysis is no longer valid, since the characteristic width of the front is comparable with the period of the structure at small scale. A more careful analysis is necessary to find an estimation in this case. (See Appendix A for details.)

The above interval is obtained because $G$ has parts independent of $y$ from a mode in (26) parallel with the $x$ axis. In principle, one might think that equivalent calculations can be performed for the type-II front, but Malomed et al. in [8] proved that pinning effects are not possible under these conditions. This argument can be generalized by imposing that a 

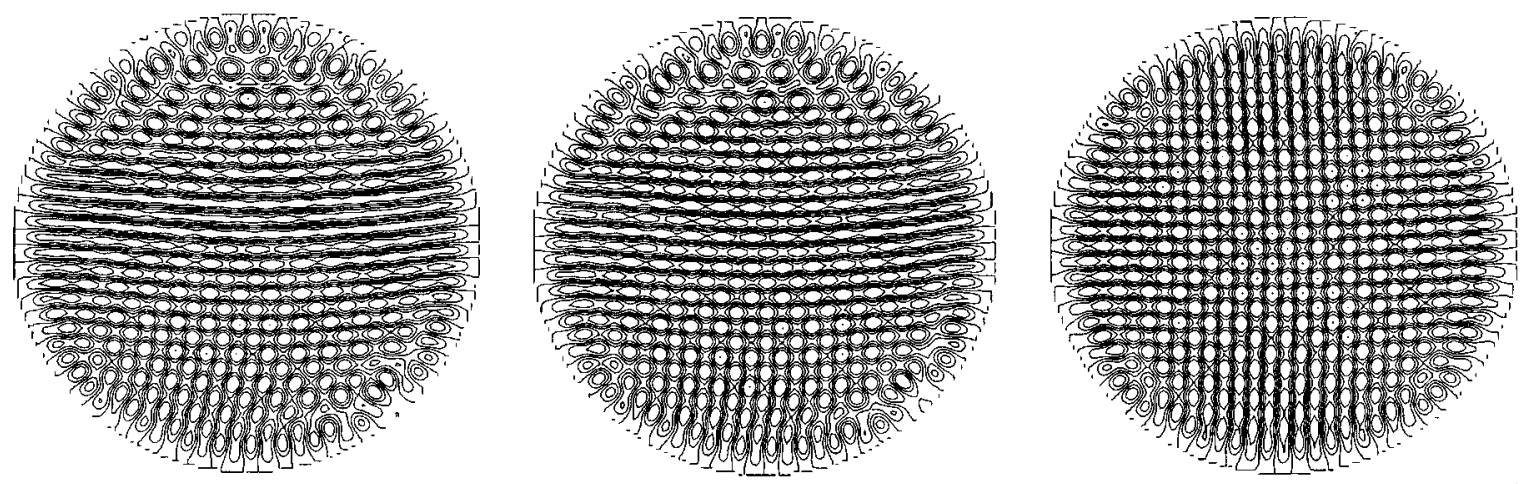

FIG. 4. A transient front at $\varepsilon=0.0497$ shown at the times $T_{1}=20000, T_{2}=60000$, and $T_{3}=70000$ correspondingly. One can see how small-scale squares are formed in the roll-dominated front, eventually leading to destabilization of the front.

dependence on $y$ close to 0 in $G$ is necessary for pinning. The $y$ dependence in our case takes the form $\sin \left(k_{c} L \theta\right)$, where $k_{c} L \theta \ll 1$, i.e., that the pinning may be seen for $\theta \ll\left(k_{c} L\right)^{-1}$, where $L$ is the system length in the $y$ direction.

\section{NUMERICAL RESULTS}

In order to simulate the evolution of the GSHE, we used a pseudospectral method and a semi-implicit time integration scheme in a $128 \times 128$ point grid. All the following results have been obtained with parameter values: $\alpha=0.036$, $\gamma=0.24$, and $\Delta t=1$ [in units of Eq. (2)]. We have modeled a circular dish with an aspect ratio $\Gamma$ defined as the quotient between the diameter, $D=128 D X=128 \pi / 4=32 \pi$ and the depth of the box $d$. In this case, we have $|\mathbf{k}|=1$, which leads to a wavelength of $\lambda=2 \pi /|\mathbf{k}|=2 \pi$. Therefore, we have $\Gamma$ $=D / d=32$. The BC's used are those commented in Sec. II ( $\psi=0$ and $\partial_{\mathbf{n}} \psi=0$ on the sidewalls). All two-dimensional (2D) plots are contour plots. From [4] we have set $c=3$, which gives $\beta=-0.28, a=0.072, b=3.12, d=5.04$, and $e=2.16$. With these values we get $g_{1}=b / c=1.04, g_{2}=1.68$, and $g_{3}=0.72$, which are values in good agreement with those used in Sec. III. Calculating $\left(2 g_{1}+1\right) /\left(2 g_{2}+g_{3}\right)$ we get $3.08 / 4.08<1$, showing that we are dealing with the full stability interval of the hexagons.

In Sec. III we used the Lyapunov functional $F[A]$ of the amplitude equations (10). But the GSHE equation (and BC's) also has a potential (Lyapunov functional) $F[\psi]$ of the form

$$
\begin{aligned}
F[\psi]= & \int d x d y\left\{-\frac{\varepsilon}{2} \psi^{2}-\frac{\alpha}{3} \psi^{3}+\frac{\beta}{4} \psi^{4}+\frac{1}{2}[(\Delta+1) \psi]^{2}\right. \\
& \left.+\frac{\gamma}{4} \psi^{2} \Delta^{2} \psi^{2}\right\}
\end{aligned}
$$

a feature that can be used to address some general statements on possible solutions of the GSHE.

\section{A. Coexisting solutions and sidewall effects}

It is easily realized that the circular finite boundary must have an effect on the results obtained. The solutions are forced to contain defects because neither hexagons nor squares can fill a circle without defects. Our first aim is to determine numerically the parameter range of stability of both hexagons and squares, i.e., find the range in which we may find pinning. This has been done by taking an initially imposed pattern of hexagons and determining the minimal and maximal values of $\varepsilon$ for which they are stable. Thereafter, this range of parameter values has been studied with an initial pattern of squares. The result is $\varepsilon_{s}=0.045 \leqslant \varepsilon$ $\leqslant 0.13=\varepsilon_{h}$. In these simulations we obtain stable hexagonal pattern at parameter values where $F[\psi]$ has the lower value for squares. It has earlier been argued that in this case the squares would invade the hexagons [14], but this is only true for systems where both structures are present, (see e.g., Fig. 4). Furthermore, it has been shown that in a range just below the above-mentioned values, the hexagons are stable, and directly above, the squares are stable. The transition is therefore hysteretic (see Fig. 1) as we previously found analytically.

An important feature of a positive defined Lyapunov functional is that for a given solution of the system, it is an ever constant or decreasing function of time. This mathematical result has an experimental interpretation as well. As is known from the literature [25] the presence of defects (which as earlier stated are unavoidable in our system) contributes by increasing the Lyapunov functional. The evolution of the system will eliminate defects and thereby lower the Lyapunov functional until a stationary state is reached. This means that the only stable solutions of the equation are local minima of the functional or metastable solutions, but since we are working with a numerical approximation the latter is not possible due to unavoidable perturbations. Furthermore, the sidewalls are known to be responsible for wave-number selection mechanisms, etc.

Due to sidewall effects, a front moves faster in a finite system than in an infinite one. In a moving front we get rolls that are far from perpendicular to the sidewalls. These rolls, together with those on the other side of the boundaries, form squarelike structures. $\left(\nabla_{n} \psi=0\right.$ is a symmetric, reflective BC). This means that we have squarelike defects on the boundaries in a front moving from a structure of squares invading a hexagonal region. As shown in Ref. [23] this type of defect helps to increase the velocity of the transition. The effect on the interval of pinning is very complicated. The boundaries help stabilize the rolls of the front, thereby widening the interval, but the presence of squarelike defects at the bound- 

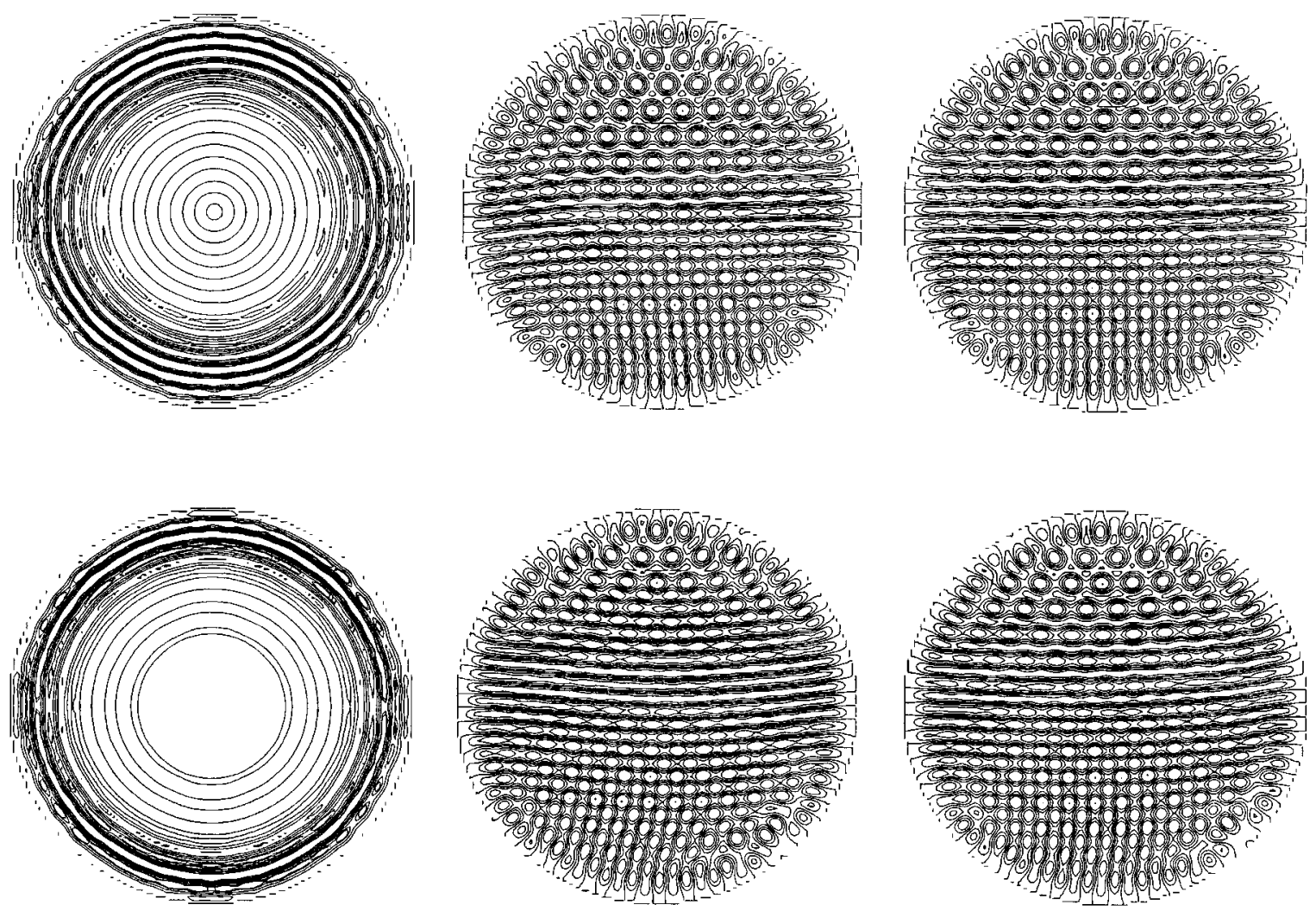

FIG. 5. A pinned front for $\varepsilon=0.04845$ (top) shown at the times $T_{1}=15, T_{2}=10000$, and $T_{3}=50000$ and for $\varepsilon=0.0495$ (bottom) shown at the times $T_{1}=10, T_{2}=20000$, and $T_{3}=100000$. Note the rolls in the front area and squarelike patterns near the boundaries in the hexagonal region.

aries of the hexagonal region (see Fig. 5) will most likely have the opposite effect.

We will search in Sec. IV B for solutions of the system containing a front with zero velocity, i.e., a pinning effect [8]. Such a solution may appear when the two potential minima have (almost) equal values.

\section{B. Pinning effects}

In a previous section we showed that the pinning is possible for an interval of $\varepsilon$. By using a low constant amplitude initial surface for the simulation of the GSHE we found pinning effects at least between $0.04845 \leqslant \varepsilon \leqslant 0.0495$. The whole interval may be larger, since we took only a few initial conditions, and the basin of attraction of the pinned front can be wider. If we compare the length of this interval for $\varepsilon=0.01$ in Eq. (28), we find that $C_{3}=0.0017$. This can be considered $O(\epsilon)$. Pinned fronts of type I can be observed in Fig. 5, for the two border values of $\varepsilon$. The system has also been run using a $256 \times 256$ grid. In this case the fronts formed in a different direction, but apart from this, they formed similarly to the ones shown.

We would like to point out that at times it is necessary to watch out for very long transients. In Fig. 4 something similar to a stable pinned front appears as far as $T=60000$, but at $T=70000$ the front is gone and the squares have invaded the whole surface. From this we can infer that the small-scale structures slowly form squares in the area dominated by un- derlying rolls, and that this process slowly eliminates the stability of the pinning.

In Fig. 6 we have initialized our system with the two types of fronts, I and II, in order to compare their stability. From the figures it is very clear that the type-II front is not pinned, and is indeed rather unstable. This confirms the analytical conclusion that the front has to be close to perpendicular to one of the modes in both structures in order to possibly be pinned.

A scheme of the interacting modes in a pinned front is presented in Fig. 3. For the type-I front one can easily deduce that in the region of the front the disappearing mode of the squares is the parallel one. The perpendicular one exists in both squares and hexagons and by looking at, e.g., Fig. 5 one realizes that it indeed also exists in the front. The same result has been obtained from the analytical calculations, where a front is obtained without changing the four interacting modes [indeed, a mode may exist in the front $(A \neq 0)$ ]. This is a roll mode, which under the conditions considered here is unstable. In this there is no contradiction, and actually this is in good agreement with earlier results, where a roll survives in the typical defect (penta-hepta pair) in a hexagonal pattern [23].

For values of $\varepsilon$ just outside the pinning interval, the velocity of the front (which we estimate as the velocity with which the domain of hexagons decreases) is rather large, in the sense that the transition is an abrupt one. 

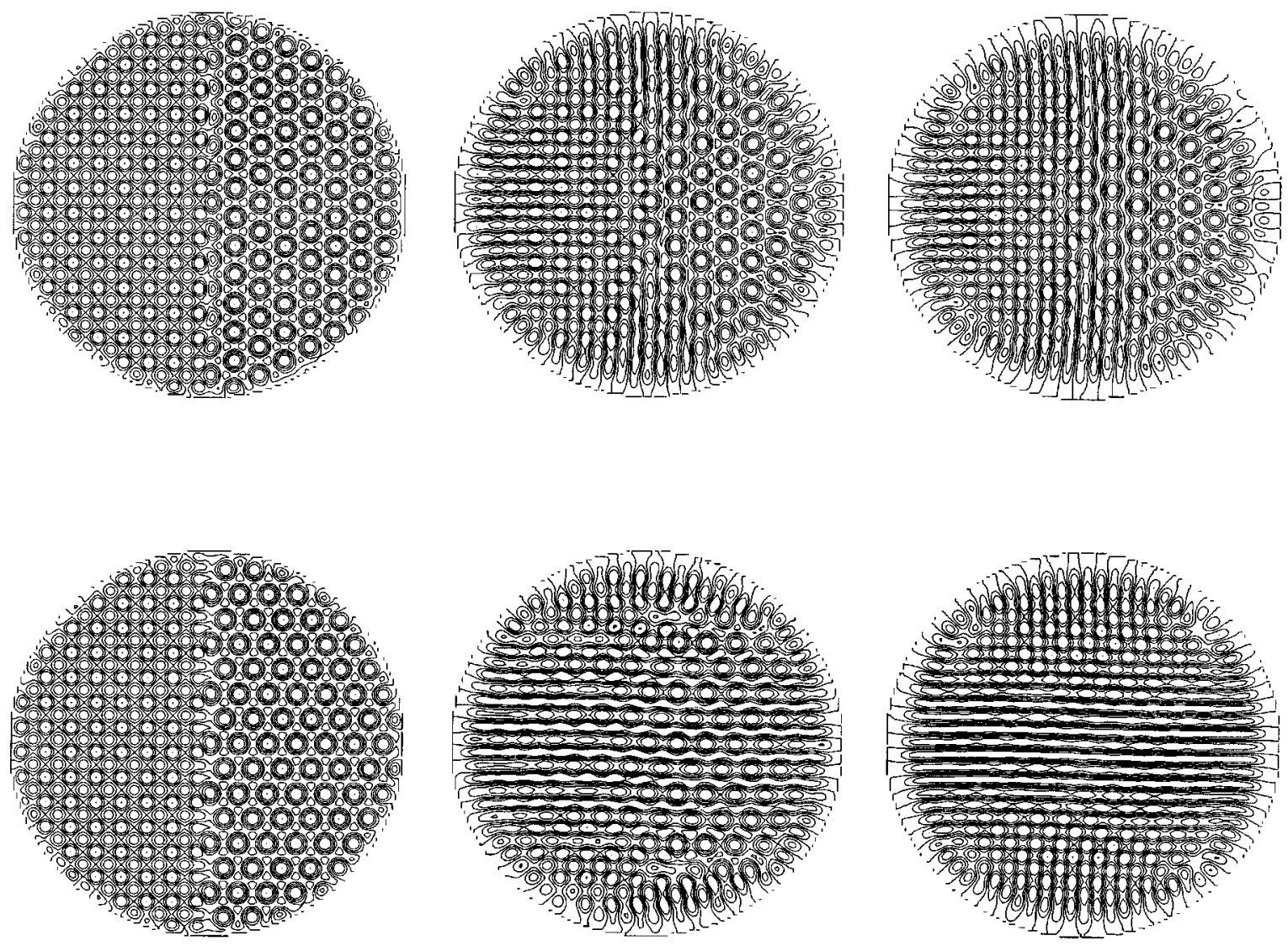

FIG. 6. Fronts of type I (top) and type II (bottom) at $\varepsilon=0.049$ shown at the times $T_{1}=1, T_{2}=250$, and $T_{3}=800$, respectively. The type-I front is clearly stable and rolls are forming in the front. In the lower evolution series the square pattern will slowly overtake the whole surface.

\section{CONCLUSIONS}

In the present paper we have studied the competition between hexagons and squares in a generalized SwiftHohenberg equation. This equation has been proposed as a model for Bénard-Marangoni convection and for ferrofluids under the influence of a vertical magnetic field. Using the model, we have derived amplitude equations and we have performed a stability analysis under homogeneous perturbations. A Lyapunov functional has been found for the amplitude equations, and with it a potential was derived. Using this we calculated a value of the control parameter for which our hexagon-square front is pinned. A thorough analysis of fronts between two structures containing more than one mode (here three and two) has been reported. This analysis has been enhanced taking into account the nonadiabatic effects of the small scales. The pinning is possible in a whole parameter interval, and we have provided a further criterion necessary to see pinning: The fronts need to be perpendicular to a fundamental mode of both competing patterns in order to be stable.

The analytical study is in agreement with numerical simulations of GSHE. In this analysis, a Lyapunov functional for the generalized Swift-Hohenberg equation has also been found, insuring the relaxational nature of a system with infinite extent. This numerical study has demonstrated the great influence of the sidewalls. Fronts have indeed been found in an interval of parameter values, and it has been shown that these fronts consist of rolls, though such solutions are unstable in our system. Proof of very long transients in systems close to pinning conditions have been shown as well.

All these results are in qualitative agreement with recent experimental observations by Nitschke and Thess [6] and Schatz [7] on Bénard-Marangoni convection and by Wesfreid [4] on interfacial instabilities in ferrofluids, where hexagons and squares coexist for a full range of the control parameter. Even though sidewall effects are crucial in explaining the patterns observed in these experiments [6], these effects do not prevent the obtaining of stable type-I fronts between squares and hexagons. Some work is planned in order to make a closer comparison with these results.

\section{ACKNOWLEDGMENTS}

We benefited from discussions with M. Bestehorn, M. Schatz, A. Thess, and M. Tribelsky. We acknowledge partial financial support from DGICYT (Spanish Government) under Grant No. PB93-0708. This project has been supported by the ERASMUS programs, ICP: 94-B-1028/13.

\section{APPENDIX A}

In this appendix we calculate analytically the value of the control parameter $\delta_{1}$ to have a stable front. This can be per- 
formed with the following hypotheses: $0<g_{1}-1 \ll 1, g_{2}-1$ small, and by taking $g_{3}=\frac{3}{4}+g_{2}$ in Eqs. (16), $\theta=0$, then $D_{2}=D_{3}$. We calculate a first integral of the system that will represent a constant energy, something necessary to obtain pinned fronts:

$$
\begin{aligned}
E= & L-\sum_{j=1}^{6} a_{j}^{\prime} \frac{\partial L}{\partial a_{j}^{\prime}} \\
= & -\frac{1}{2} a_{1}^{\prime 2}-\frac{1}{4} a_{2}^{\prime 2}-\frac{\delta}{2} a_{1}^{2}-\delta a_{2}^{2}-\frac{\delta}{2} a_{4}^{2} \\
& +\frac{1}{4} a_{1}^{4}+\frac{1}{2} a_{2}^{4}+\frac{1}{4} a_{4}^{4}+\frac{g_{1}}{2}\left(2 a_{2}^{2} a_{1}^{2}+a_{2}^{4}\right)+\frac{g_{2}}{2}\left(a_{4}^{2} a_{1}^{2}\right) \\
& +g_{3} a_{2}^{2} a_{4}^{2}-a_{1} a_{2}^{2} .
\end{aligned}
$$

In order to represent the spatial dependence of (16) in a more adequate way we perform the following transformations:

$$
\begin{gathered}
a_{1}=\frac{2 r \cos \chi}{\sqrt{3}\left(g_{1}-1\right)}, \quad a_{2}=a_{3}=\left(\frac{2}{3}\right)^{1 / 2} \frac{r \sin \chi}{g_{1}-1}, \\
a_{4}=\frac{r}{g_{1}-1} \sqrt{2\left(\cos ^{2} \chi-\frac{1}{3}\right)}, \quad z=\frac{2}{\sqrt{3\left(g_{1}-1\right)}} X^{\prime},
\end{gathered}
$$

where $r=1+O\left(g_{1}-1\right)$. (In the transformation of $a_{4}$ we have used the BC's $a_{4}=0$ at $z \rightarrow-\infty$ and $a_{4}=a_{1}$ at $z \rightarrow \infty$.) We introduce (A2) in (A1) and find that the extra amplitude introduced in the calculations in fact does not affect the equation obtained in [8] for $\chi$ and corresponding BC's. For a front between hexagons and squares we get:

$$
\begin{gathered}
\frac{d \chi}{d z}=\sin \chi \frac{1-\sqrt{3} \cos \chi}{\left(4-3 \cos ^{2} \chi\right)^{1 / 2}}, \\
\chi \rightarrow \cos ^{-1}(1 / \sqrt{3}) \quad \text { when } z \rightarrow-\infty, \\
\chi \rightarrow 0 \quad \text { when } z \rightarrow \infty .
\end{gathered}
$$

The solution of this differential equation can be written in an implicit form (see Malomed et al. [8]):

$$
\begin{aligned}
z-z_{0}= & \frac{\sqrt{3}-1}{4} \ln \frac{4+3 \cos \chi+\left(4-3 \cos ^{2} \chi\right)^{1 / 2}}{1+\cos \chi} \\
& +\frac{\sqrt{3}+1}{4} \ln \frac{4-3 \cos \chi+\left(4-3 \cos ^{2} \chi\right)^{1 / 2}}{1-\cos \chi} \\
& -\frac{3}{2} \ln \frac{4 / \sqrt{3}-\cos \chi+\left(4-3 \cos ^{2} \chi\right)^{1 / 2}}{\sqrt{3} \cos \chi-1}
\end{aligned}
$$

where $z_{0}$ is a reference point. In the above calculations we have used two assumptions without explanation, namely $r=1+O\left(g_{1}-1\right) \quad$ and $\quad \delta=\delta_{2}\left(g_{1}-1\right)^{-2}=\left(4 / 3 g_{2}+\frac{2}{3}\right)\left(g_{1}\right.$ $-1)^{-2}$. We may check the validity of these assumptions $a$ posteriori: we introduce the values in expression [21] and calculate $|V(-\infty)-V(\infty)|$. For $\delta=\delta_{1}=\delta_{2}\left(g_{1}-1\right)^{-2}$ we should have pinning, i.e., we should obtain $\mid V(-\infty)$ $-V(\infty) \mid \ll 1$. An explicit calculation gives

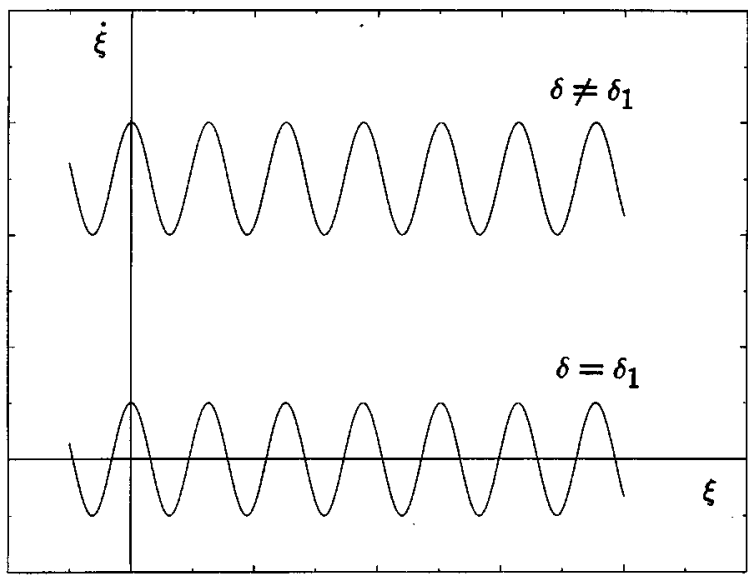

FIG. 7. Schematic behavior of the function $\dot{\xi}(\xi, \delta)$. This function $\dot{\xi}$ has (vertical) translational dependence on $\delta$, showing that we have stable solutions for a whole interval of values.

$$
\begin{aligned}
V(-\infty)-V(\infty)= & \frac{3}{2} \delta a_{h}^{2}-\frac{3}{4} a_{h}^{4}-\frac{3}{2} g_{1} a_{h}^{4}+a_{h}^{3}-\delta a_{c}^{2}+\frac{1}{2} a_{c}^{4} \\
& +\frac{1}{2} g_{2} a_{c}^{4} \\
= & {\left[\frac{2}{3} \delta_{2}-\frac{4}{27}-\frac{8}{27}-\frac{8}{27}\left(g_{1}-1\right)\right.} \\
& \left.+\frac{8}{27}\left(g_{1}-1\right)\right] \frac{1}{\left(g_{1}-1\right)^{4}} \\
& -\left[\frac{4}{3} \delta_{2}-\frac{8}{9}-\frac{8}{9} g_{2}\right] \frac{1}{\left(g_{1}-1\right)^{4}}=0,
\end{aligned}
$$

which clearly indicates that these assumptions are justified.

\section{APPENDIX B}

In this appendix we present detailed calculations to obtain the range of the control parameter for which the pinning of a front is possible. Our starting point is the perturbation equation (27). The linear operator of the left-hand side (lhs) of this equation has a null eigenfunction $\partial \psi_{0} / \partial \xi$, since $\psi_{0}$ is a solution at leading order. Equation (27) has a solution if the right-hand side (rhs) of the equation is orthogonal to this eigenfunction (solvability condition):

$$
\begin{aligned}
\frac{d \xi}{d t} \int_{-\infty}^{\infty}\left(\frac{\partial \psi_{0}}{\partial \xi}\right)^{2} d x d y= & \left(\delta-\delta_{1}\right) \int_{-\infty}^{\infty} \psi_{0} \frac{\partial \psi_{0}}{\partial \xi} d x d y \\
& +\int_{-\infty}^{\infty} G(x, y) \frac{\partial \psi_{0}}{\partial \xi} d x d y,
\end{aligned}
$$

where

$$
G=\sum_{n=1}^{4} G_{n}(X) \cos \left(\frac{n\left(\xi_{1}+X\right)}{\sqrt{\epsilon}}\right)
$$

We write some of the terms of $G_{1}$ to give an idea of its shape, but it is too cumbersome an expression to be written completely: 


$$
\begin{aligned}
G_{1}= & 2 a_{1} \delta_{1}+\alpha\left[\left(6 a_{1}^{2}+4 a_{2}^{2}+4 a_{3}^{2}+4 a_{4}^{2}\right) \frac{d a_{1}}{d X}\right. \\
& \left.+8 a_{1} a_{2} \frac{d a_{2}}{d X}+8 a_{1} a_{3} \frac{d a_{3}}{d X}+8 a_{1} a_{4} \frac{d a_{4}}{d X}\right] \\
& +\cdots .
\end{aligned}
$$

We average, once again, in the $y$ direction in this expression, and every integral that has $y$ dependence is equal to zero. Changing to slow variables in the amplitudes,

$$
X=\sqrt{\epsilon} x, \quad \xi_{1}=\sqrt{\epsilon} \xi, \quad T=\epsilon t,
$$

we get the equation

$$
\frac{d \xi_{1}}{d T}=\left\{\int_{-\infty}^{\infty}\left[\left(\frac{d a_{1}}{d X}\right)^{2}+2\left(\frac{d a_{2}}{d X}\right)^{2}+\left(\frac{d a_{4}}{d X}\right)^{2}\right] d X\right\}^{-1}\left(-\left(\delta-\delta_{1}\right) C+\sum_{n=1}^{4} \int_{-\infty}^{\infty} G_{n}(X) \cos \frac{n\left(\xi_{1}+X\right)}{\sqrt{\epsilon}} d X\right),
$$

where $C$ takes the form

$$
C=\frac{3\left(1+g_{2}\right)\left[1+2 \delta+4 \delta g_{1}+\sqrt{1+4 \delta\left(1+2 g_{1}\right)}\right]-4 \delta\left(1+2 g_{1}\right)^{2}}{2\left(1+2 g_{1}\right)^{2}\left(1+g_{2}\right)}
$$

If we consider the analytical continuation of $G(X)$ for complex $X$ and denoted by $X_{s}$ the singularity of this function closest to the real axis, then applying the theorem of the residues the latter of the integrals is

$$
\sum_{n=1}^{4} C_{n} \exp \left(-n \operatorname{Im} X_{s} / \sqrt{\epsilon}\right) \cos \left[n\left(\xi_{1}+\operatorname{Re} X_{s}\right) / \sqrt{\epsilon}\right] \text {. }
$$

(This can be done because the contribution of $X_{s}$ is the dominating part of the integral [24].) Equation (B6) can be written as

$$
\begin{aligned}
\frac{d \xi_{1}}{d T}= & C_{0}\left(\delta-\delta_{1}\right)+\sum_{n=1}^{4} C_{n} \\
& \times \exp \left(-\frac{n \operatorname{Im} X_{s}}{\sqrt{\epsilon}}\right) \cos n \frac{\xi_{1}+\operatorname{Re} X_{s}}{\sqrt{\epsilon}} .
\end{aligned}
$$

The above equation is the equation of motion of a front in the case of small $\left|\delta-\delta_{1}\right|$. The fixed points of this equation correspond to immobile fronts between hexagons and squares. The fixed points of (B9) can now easily be shown to have the following property:

$$
\left|\delta-\delta_{1}\right|<C \exp \left(-\frac{\operatorname{Im} X_{s}}{\sqrt{\epsilon}}\right)
$$

This property is easier to demonstrate with $n=1$ only and the equation $\dot{\xi}=a+b \cos (\xi)$. For this equation we have

$$
\ddot{\xi}=0 \Rightarrow \xi=m \pi, \quad m \in Z .
$$

The minimal and maximal values of $\dot{\xi}_{1}$ are $C_{0}\left(\delta-\delta_{1}\right) \pm C_{1} \exp \left(-\operatorname{Im} X_{s} / \sqrt{\epsilon}\right)$, where both constants are positive. To have pinning, the maxima have to be positive and the minima negative. The above situation is given schematically in Fig. 7, in which it can be seen that for $\delta \simeq \delta_{1}$, there exist stable solutions (i.e., points where $\xi=0$ and $\ddot{\xi}<0$ ), a similar situation occurs in the general case $n=4$. We will estimate the interval for the solution obtained in Appendix A in the case $0<g_{1}-1 \ll 1, g_{3}=\frac{3}{4}+g_{2}$ and $g_{2}-1$ small.

The singularities of (B10) are also for $\chi$ and $d \chi / d z$; since the singularity of $d \chi / d z$ is such that $4-3 \cos ^{2} \chi\left(z_{s}\right)=0$, then $\cos \chi\left(z_{s}\right)=\sqrt{4 / 3}$, and from the implicit solution for $\chi(A 5)$ we deduce:

$$
\operatorname{Im} z_{s}=\frac{\sqrt{3}-1}{4} \pi
$$

Then the interval around $\delta_{1}$ for which the front between hexagons and squares is immobile is given by

$$
\left|\delta-\delta_{1}\right|<C \exp \left[-\frac{\sqrt{3}-1}{8} \pi\left(\frac{3\left(g_{1}-1\right)}{\epsilon}\right)^{1 / 2}\right],
$$

where $C$ is a constant.
[1] M. C. Cross and P. C. Hohenberg, Rev. Mod. Phys. 65, 851 (1993)

[2] A. Gailitis, J. Fluid Mech. 82, 401 (1977).

[3] M. Silber and E. Knobloch, Physica D 30, 83 (1988).

[4] E. Wesfreid (private communication).

[5] R. E. Rosensweig, Annu. Rev. Fluid Mech. 19, 437 (1987).

[6] K. Nitschke and A. Thess (unpublished).
[7] M. Schatz (private communication).

[8] B. A. Malomed, A. A. Nepomnyashchy, and M. I. Tribelsky, Phys. Rev. A 42, 7244 (1990).

[9] H. Herrero, C. Pérez-García, and M. Bestehorn, Chaos 4, 15 (1994).

[10] A. C. Newell and J. A. Whitehead, J. Fluid Mech. 38, 279 (1969). 
[11] L. A. Segel, J. Fluid Mech. 38, 203 (1969).

[12] J. Swift and P. C. Hohenberg, Phys. Rev. A 15, 319 (1977).

[13] M. Bestehorn and H. Haken, Phys. Rev. A 42, 7195 (1990).

[14] Y. Pomeau, Physica D 23, 3 (1986).

[15] H. Haken, Synergetics: An Introduction, 2nd ed. (SpringerVerlag, Berlin, 1978); Advanced Synergetics (Springer-Verlag, Berlin, 1983).

[16] R. Friedrich, M. Bestehorn, and H. Haken, Int. J. Mod. Phys. B 4, 365 (1990).

[17] M. Bestehorn and H. Haken, Z. Phys. B 57, 329 (1984).

[18] M. Bestehorn and C. Pérez-García, Physica D 61, 67 (1992).

[19] The two sets of variables are introduced by substituting $\mathbf{x}$ with $\mathbf{x}+\epsilon \mathbf{X}$ and $t$ by $t+\epsilon^{2} T$.

[20] M. Bestehorn, Phys. Rev. E 48, 3622 (1993).

[21] S. Lauzeral, S. Metens, and D. Walgraef, Europhys. Lett. 24, 707 (1993).

[22] This is easily realized by noting that for instance $\partial \dot{A}_{j} / \partial A_{k} \propto A_{k}$ for $j=1,2,3$ and $k=4,5,6$. This term vanishes in the Jacobian for hexagons, since in this case $A_{k}=0$.

[23] E. Pampaloni, C. Pérez-García, L. Albavetti, and S. Ciliberto, J. Fluid Mech. 234, 393 (1992).

[24] D. Bensimon, B. I. Shraiman, and V. Croquette, Phys. Rev. E 38, 5461 (1988).

[25] M. C. Cross, Phys. Rev. A 25, 1065 (1982). 